\title{
High-Performance Liquid Chromatography-Mass Spectrometry Profiling of Phenolic Compounds for Evaluation of Olive Oil Bitterness and Pungency
}

\author{
Georg Dierkes, ${ }^{\dagger} \|$ Sonja Krieger, ${ }^{\ddagger}$ Roman Dück, ${ }^{\ddagger}$ Annette Bongartz, ${ }^{\S}$ Oliver J. Schmitz, ${ }^{\ddagger}$ \\ and Heiko Hayen ${ }^{* \dagger}$ \\ ${ }^{\dagger}$ Department of Food Chemistry, University of Wuppertal, Wuppertal, Germany \\ ${ }^{\ddagger}$ Institute for Pure and Applied Mass Spectrometry (iPAMS), University of Wuppertal, Wuppertal, Germany \\ ${ }^{\S}$ School of Life Sciences and Facility Management, Zurich University of Applied Sciences, Grüental, Wädenswil, Switzerland
}

Supporting Information

\begin{abstract}
Bitterness and pungency are important parameters for olive oil quality. Therefore, two instrumental methods for evaluation of these taste attributes were developed. The first one is based on the photometric measurement of total phenolic compounds content, whereas the second one is based on the semiquantitative evaluation of hydrophilic compounds by highperformance liquid chromatography-mass spectrometry (HPLC-MS). Evaluation of total phenolic compounds content was performed by a modified method for the determination of the $K_{225}$ value using a more specific detection based on the $\mathrm{pH}$ value dependency of absorbance coefficients of phenols at $\lambda=274 \mathrm{~nm}$. The latter method was not suitable for correct prediction, because no significant correlation between bitterness/pungency and total phenolic compounds content could be found. For the second method, areas of 25 peaks detected in 54 olive oil samples by a HPLC-MS profiling method were correlated with the bitterness and pungency by partial least-squares regression. Six compounds (oleuropein aglycon, ligstroside aglycon, decarboxymethyl oleuropein aglycon, decarboxymethyl ligstroside aglycon, elenolic acid, and elenolic acid methyl ester) show high correlations to bitterness and pungency. The computed model using these six compounds was able to predict bitterness and pungency of olive oil in the error margin of the sensory evaluation $( \pm 0.5)$ for most of the samples.
\end{abstract}

KEYWORDS: olive oil, sensory quality, bitterness, pungency, LC-MS, partial least-squares regression

\section{INTRODUCTION}

High-quality olive oil is characterized not only by the characteristic green and fruity odor notes but also by a well observable bitter and pungent taste. Whereas the aroma of olive oil is based on the presence of volatile aroma-active compounds, the bitter and pungent taste is caused by nonvolatile hydrophilic compounds. ${ }^{1}$ The latter compounds are mainly hydrolysis products of oleuropein and ligstroside, two secoiridoid glucosides characteristic of the Oleaceae. These compounds are also responsible for the relative high resistance of virgin olive oil against oxidative spoilage and play an important role in the health benefits, which are commonly associated with virgin olive oil in the "Mediterranean diet" concept. $^{2}$

In this paper, the influence of hydrophilic phenols on organoleptic properties of virgin olive oil is studied. The bitterness and pungency intensities are parameters for the sensory evaluation of olive oil quality, the so-called "Panel Test" developed by the International Olive Council. ${ }^{3}$ Sensory results performed by well-trained panelists are reproducible and comparable with other panels. The disadvantages of sensory quality evaluation are (i) the lack of stable and standardized reference oils with different intensities of bitterness and pungency and (ii) the large number of panelists that is needed for statistically confirmed results. ${ }^{4}$
Several investigations in identifying hydrophilic phenols in olive oil were carried out in the past, ${ }^{5-10}$ but only in a few studies, an instrumental method was developed as an alternative to sensory evaluation for olive oil quality: The most common method is the determination of the so-called $K_{225}$ value developed by Gutiérrez Rosales et al. ${ }^{11}$ Calculation of the $K_{225}$ value is based on the absorbance at $\lambda=225 \mathrm{~nm}$ of the polar extract from olive oil obtained by solid-phase extraction. Beltrán et al., ${ }^{12}$ Mateos et al., ${ }^{13}$ and Inarejos-Garcia et al. ${ }^{14}$ developed alternative methods for the determination of the $K_{225}$ value, which are more selective for phenolic compounds. Selectivity improvements were achieved by using colorimetric detection after reaction with the Folin-Ciocalteau reagent, photometric detection combined with a $\mathrm{pH}$ gradient, or fluorimetric detection. Other methods for instrumental evaluation of olive oil bitterness are based on quantification of the main hydrophilic phenols oleuropein aglycon, ligstroside aglycon, decarboxymethyl oleuropein aglycon, and decarboxymethyl ligstroside aglycon in olive oil by high-performance liquid chromatography (HPLC). ${ }^{9,15-17}$ The authors of these studies obtained inconsistent results with respect to the

Received: November 2, 2011

Revised: July 16, 2012

Accepted: July 17, 2012

Published: July 17, 2012 
influence of the different phenols. The difficulty in linking dedicated compounds to the taste attributes bitternesss and pungency is highlighted in a recent perspective article: "There is still controversy about which individual phenols are the main contributors to taste attributes." 4 It is still not clear which compounds are mainly responsible for the bitter taste and how they have to be weighted in regression models. Furthermore, there is only limited knowledge about the correlation between the pungent taste and the contents of hydrophilic compounds in olive oil. Andrewes et al. identified the decarboxymethyl ligstroside aglycon as a pungent compound in olive oil, but correlation between quantitative data and sensory data was not verified. $^{18}$

In this work, we show that evaluation of bitterness and pungency of olive oils from different varieties and region of origin is not possible by determination of the total phenolic content. Even quantification of single hydrophilic phenols does not result in a reliable prediction model for bitterness and pungency and is no alternative to the photometric determination of total phenolic components. The main goal of this study was to set up a HPLC-high-resolution-MS profiling method to screen for additional potential bitter or pungent hydrophilic compounds. Thus, a profiling method in combination with sensory data and statistical analysis should be a helpful tool for identifying all relevant bitter and pungent components and should give new evidence for alternative approaches to set up prediction models for bitterness and pungency in olive oil.

\section{MATERIALS AND METHODS}

Reagents and Materials. Chemicals. The following compounds were obtained commercially: 3-hydroxytyrosol, tyrosol, 4-hydroxyphenyl acetic acid, acetic acid, boric acid, and orthophosphoric acid from Aldrich (Steinheim, Germany). HPLC-grade (supra gradient grade) acetonitrile was purchased from Bischoff Chromatography (Leonberg, Germany). Formic acid was of analytical grade ( $98 \%)$ and purchased from Fluka (Steinheim, Germany). Water was purified using a TKA ultrapure water system (Thermo Fisher Scientific, Niederelbert, Germany). Briton-Robinson buffer preparation was as follows: $5 \mathrm{mmol}$ of acetic acid $(300 \mathrm{mg}), 5 \mathrm{mmol}$ of boric acid (309 $\mathrm{mg}$ ), and $5 \mathrm{mmol}$ of orthophosphoric acid $(490 \mathrm{mg}$ ) in $1 \mathrm{~L}$ of water, adjusted to $\mathrm{pH} 5$ with $1 \%$ sodium hydroxide solution.

Olive Oils. Fifty-four monovarietal and multivarietal olive oil samples were from different countries [18 Spain, 15 Italy, 10 Portugal, 4 Israel, 3 Greece, 2 California (United States), 1 France, and 1 Turkey]. The varieties of the used drupes are provided in the Supporting Information. The oil samples were stored at $-20{ }^{\circ} \mathrm{C}$ until use.

Sensory Analyses. Sensory evaluations were performed by the Swiss Olive Oil Panel (expert olive oil panel of the Zurich University of Applied Sciences) consisting of at least 8-10 assessors. All oils were subjected to an extended panel test based on EU Regulation 640/2008 [Commission Regulation (EC), No. 640/2008 of 4 July 2008 amending Regulation (EEC) No. 2568/91 on the characteristics of olive oil and olive-residue oil and on the relevant methods of analysis, 2008] as well as the IOC (International Olive Council) instructions for the objective assessment of olive oils (COI/T.20/Doc.No.15/ Rev.1). The results are expressed as the median of the rating of at least eight persons. The robust mean coefficient of variation was in all cases $<20 \%$.

Photometric Determination of Total Phenol Content. A combined method of the procedures described by Beltrán et al. ${ }^{12}$ and Mateos et al. ${ }^{13}$ was used. Beltrán et al. determined the total phenol content by spectrometric detection at $\lambda=225 \mathrm{~nm}$ after isolation of the hydrophilic phenols. Mateos et al. developed a method for the direct determination of total phenols in olive oil using a $\mathrm{pH}$ gradient $(5-13)$ and spectrometric detection at $\lambda=274 \mathrm{~nm}$. This method is more selective for phenols, but there are solubility problems with sodium hydroxide, which is used for modifying the $\mathrm{pH}$ value of the solution of olive oil in 1-propanol. Therefore, a combined method using selective detection of total phenol contents at two different $\mathrm{pH}$ values $(5$ and 13) at $\lambda=274 \mathrm{~nm}$ after isolation was set up. This detection is more selective than the detection at $\lambda=225 \mathrm{~nm}$, and after isolation, there are no solubility problems with sodium hydroxide in the polar extraction medium. Isolation of the hydrophilic compounds was performed by liquid-liquid extraction, because solid-phase extraction gives no better recovery at higher costs. ${ }^{14}$

A $0.1 \mathrm{~g}$ amount of olive oil was dissolved in $3 \mathrm{~mL}$ of pentane and extracted with $5 \mathrm{~mL}$ of methanol:Briton-Robinson buffer ( $\mathrm{pH} 5.2$ ) (80:20, v:v). The absorbance of the polar phase was measured at $\lambda=$ $274 \mathrm{~nm}$. After this, the $\mathrm{pH}$ of the solution was adjusted to $\mathrm{pH} 13$ by adding $150 \mu \mathrm{L}$ of aqueous sodium hydroxide solution $(1 \mathrm{~g} / 100 \mathrm{~mL})$. The absorbance of this solution was measured again at $\lambda=274 \mathrm{~nm}$. The measure total phenol, which is a degree for the content of all summarized phenols in $1 \mathrm{~g}$ of olive oil, is calculated by the following formula:

$$
\text { total phenol }=\frac{A_{2}-A_{1}}{m_{\text {sample }}}
$$

with $A_{1}$, absorbance at $\mathrm{pH} 5 ; A_{2}$, absorbance at $\mathrm{pH} 13$; and $m_{\text {sample }}$, weighted sample.

Extraction of Hydrophilic Compounds. A $1.0 \mathrm{~g}$ amount of olive oil was dissolved in $5 \mathrm{~mL}$ of pentane and after adding $100 \mu \mathrm{L}$ of internal standard solution (4-hydroxyphenyl acetic acid, $1 \mathrm{mg} / \mathrm{mL}$ in methanol) extracted with $5 \mathrm{~mL}$ of methanol:water (80:20, v:v). The pentane phase was discarded, and the polar phase was extracted with 3 $\mathrm{mL}$ of pentane for another time. The polar phase was directly used for HPLC-MS analysis.

HPLC-MS. The HPLC analysis was performed on an Agilent series 1290 liquid chromatographic system equipped with a 6538 UHD Accurate Mass Q-TOF. A pentafluorophenyl modified silica gel column [Luna $\mathrm{PFP}(2), 3 \mu \mathrm{m}, 100 \AA$, $150 \mathrm{~mm} \times 2.0 \mathrm{~mm}$ ] (Phenomenex, Aschaffenburg, Germany) maintained at $30{ }^{\circ} \mathrm{C}$ was used. Elution was performed at a flow rate of $0.3 \mathrm{~mL} / \mathrm{min}$, using a mobile phase of $0.1 \%$ formic acid (solvent A) and a mixture of acetonitrile $/ 0.1 \%$ formic acid (90:10, v:v) (solvent B). The solvent gradient changed according to the following conditions: from 10 to $40 \%$ B in $5 \mathrm{~min}$, to $50 \%$ B in $3 \mathrm{~min}$, to $90 \%$ B in $2 \mathrm{~min}, 90 \%$ B hold for $4 \mathrm{~min}$, to $10 \% \mathrm{~B}$ in $2 \mathrm{~min}$, and reequilibration of the column for $5 \mathrm{~min}$. The injection volume was $3 \mu \mathrm{L}$.

The HPLC system was connected to a time-of-flight mass spectrometer Agilent 6538 UHD Accurate Mass Q-TOF (Agilent Technologies, Santa Clara, CA) equipped with an electrospray interface operating in the negative ion mode, using the following conditions: capillary voltage, $4000 \mathrm{~V}$; nebulizer pressure, $40 \mathrm{psi}$; drying gas flow rate, $10 \mathrm{~L} / \mathrm{min}$; gas temperature, $300{ }^{\circ} \mathrm{C}$; skimmer voltage, 50 $\mathrm{V}$; octapole rf, $150 \mathrm{~V}$; and fragmentor voltage, $130 \mathrm{~V}$. LC-MS accurate mass spectra were recorded across the range of $\mathrm{m} / z$ 50-1000. The instrument performed the internal mass calibration automatically, using a dual-nebulizer electrospray source with an automated calibrant delivery system, which introduces the flow from the outlet of the chromatograph together with a low flow (approximately $5 \mu \mathrm{L} / \mathrm{min}$ ) of a calibrating solution, which contains the internal reference masses $\mathrm{m} / z 119.03632$ and $\mathrm{m} / z 966.000725$. Optimal ionization conditions were evaluated by infusing $10 \mathrm{mg} / \mathrm{L}$ of 3-hydroxytyrosol in a mixture of methanol $/ 0.1 \%$ formic acid (50:50, v:v). The instrument provided a resolution of at least $10000(\mathrm{~m} / z$ 302). MS/MS spectra were obtained in negative ion mode. Fragmentation was performed using a fixed collision energy of $10 \mathrm{eV}$. The full-scan data were processed with Agilent Mass Hunter software version B.04.00.

Semiquantitative Analysis. 4-Hydroxyphenyl acetic acid was selected as an internal standard (IS) for semiquantitative analysis by HPLC-MS. This compound possesses structural similarity with the analytes and was already successfully applied in secoiridoid derivative quantification. ${ }^{9,16}$ The analytes were quantified by assessing the peak 
Table 1. Hydrophilic Compounds Detected by HPLC-MS Profiling Method in Olive Oil

\begin{tabular}{|c|c|c|c|c|c|c|}
\hline no. & retention time $(\mathrm{min})$ & possible components & $m / z \operatorname{expt}$ & formula & error (ppm) & ref \\
\hline 1 & 3.19 & hydroxytyrosol $^{a}$ & 153.0556 & $\mathrm{C}_{8} \mathrm{H}_{9} \mathrm{O}_{3}$ & 0.8 & 10 \\
\hline 2 & 5.82 & azelaic $\operatorname{acid}^{a}$ & 187.0976 & $\mathrm{C}_{9} \mathrm{H}_{15} \mathrm{O}_{4}$ & -0.1 & 10 \\
\hline $3 a$ & 5.55 & desoxy elenolic acid $1^{b}$ & 225.0763 & $\mathrm{C}_{11} \mathrm{H}_{13} \mathrm{O}_{5}$ & 2.4 & $6^{c}$ \\
\hline $3 b$ & 6.31 & desoxy elenolic acid $2^{b}$ & 225.0768 & $\mathrm{C}_{11} \mathrm{H}_{13} \mathrm{O}_{5}$ & 0.2 & $6^{c}$ \\
\hline 4 & 12.34 & myristic acid ${ }^{a}$ & 227.2018 & $\mathrm{C}_{14} \mathrm{H}_{27} \mathrm{O}_{2}$ & -0.7 & 10 \\
\hline 5 & 6.23 & elenolic acid ${ }^{a}$ & 241.0723 & $\mathrm{C}_{11} \mathrm{H}_{13} \mathrm{O}_{6}$ & -2.2 & 10 \\
\hline $6 \mathrm{~b}$ & 7.75 & elenolic acid methylester $2^{b}$ & 255.0874 & $\mathrm{C}_{12} \mathrm{H}_{15} \mathrm{O}_{6}$ & 0.1 & $14^{c}$ \\
\hline 7 & 8.85 & apigenin $^{a}$ & 269.0460 & $\mathrm{C}_{15} \mathrm{H}_{9} \mathrm{O}_{5}$ & -1.7 & 10 \\
\hline 8 & 7.66 & luteolin $^{a}$ & 285.0412 & $\mathrm{C}_{15} \mathrm{H}_{9} \mathrm{O}_{6}$ & -2.6 & 10 \\
\hline 9 & 9.11 & chrysoeriol $^{a}$ & 299.0554 & $\mathrm{C}_{16} \mathrm{H}_{11} \mathrm{O}_{6}$ & 2.4 & 10 \\
\hline 10 & 8.06 & decarboxymethyl ligstroside aglycon $^{a}$ & 303.1245 & $\mathrm{C}_{17} \mathrm{H}_{19} \mathrm{O}_{5}$ & -2.3 & 6 \\
\hline 11 & 6.91 & decarboxymethyl oleuropein aglycon $^{a}$ & 319.1193 & $\mathrm{C}_{17} \mathrm{H}_{19} \mathrm{O}_{6}$ & -1.8 & 6 \\
\hline $12 \mathrm{a}$ & 4.89 & decarboxymethyl $10-\mathrm{OH}$-oleuropein aglycon $1^{a}$ & 335.1139 & $\mathrm{C}_{17} \mathrm{H}_{19} \mathrm{O}_{7}$ & -0.8 & 10 \\
\hline 13 & 7.85 & pinoresinol $^{a}$ & 357.1333 & $\mathrm{C}_{20} \mathrm{H}_{21} \mathrm{O}_{6}$ & 3.0 & 10 \\
\hline 14 & 10.25 & dehydro ligstroside aglycon ${ }^{b}$ & 359.1144 & $\mathrm{C}_{19} \mathrm{H}_{19} \mathrm{O}_{7}$ & -2.1 & \\
\hline $15 a-i$ & $6.51-10.59$ & ligstroside aglycon $1-9^{a}$ & 361.1303 & $\mathrm{C}_{19} \mathrm{H}_{21} \mathrm{O}_{7}$ & -2.8 & 6 \\
\hline 16 & 9.14 & dehydro oleuropein aglycon ${ }^{b}$ & 375.1097 & $\mathrm{C}_{19} \mathrm{H}_{19} \mathrm{O}_{8}$ & -3.1 & \\
\hline $17 a-i$ & $5.79-9.52$ & oleuropein aglycon $1-9^{a}$ & 377.1254 & $\mathrm{C}_{19} \mathrm{H}_{21} \mathrm{O}_{8}$ & -3.2 & 6 \\
\hline $18 \mathrm{a}$ & 6.01 & methyl oleuropein aglycon $1^{a}$ & 391.1399 & $\mathrm{C}_{20} \mathrm{H}_{23} \mathrm{O}_{8}$ & -0.2 & 10 \\
\hline $18 b$ & 9.11 & methyl oleuropein aglycon $2-8^{a}$ & 391.1399 & $\mathrm{C}_{20} \mathrm{H}_{23} \mathrm{O}_{8}$ & -0.2 & 10 \\
\hline 19 & 8.06 & acetoxypinoresinol $^{a}$ & 415.1412 & $\mathrm{C}_{22} \mathrm{H}_{23} \mathrm{O}_{8}$ & -3.3 & 10 \\
\hline $20 a$ & 7.50 & ligstroside aglycon + methanol + formic acid ${ }^{b}$ & 439.1618 & $\mathrm{C}_{21} \mathrm{H}_{27} \mathrm{O}_{10}$ & -1.9 & \\
\hline $20 b$ & 7.88 & ligstroside aglycon + methanol + formic acid $b$ & 439.1618 & $\mathrm{C}_{21} \mathrm{H}_{27} \mathrm{O}_{10}$ & -1.9 & \\
\hline
\end{tabular}

${ }^{a}$ Identification based on accurate mass and literature data. ${ }^{b}$ Tentative identification by accurate mass and MS/MS spectra. ${ }^{c}$ Detected but not identified in respective literature.

area ratios of each analyte versus that of the IS using $[\mathrm{M}-\mathrm{H}]^{-}$ions listed in Table 1. Peak resolutions were not good between all of the isomers, because of interconversion during analysis. Therefore, peak areas were determined as the sum parameter over all detected isomers of the analytes $15 \mathbf{a}-\mathbf{i}, 17 \mathbf{a}-\mathbf{i}$, and $18 \mathbf{b}$ by integration over all signals with the same $m / z$ value in a range of $\pm 10 \mathrm{ppm}$ (cf. Figure 1). The obtained quantification data have to be considered semiquantitative, because the different response factors of analytes were not determined due to the absence or limited availability of standard compounds. Furthermore, coelution may have resulted in ionization suppression or enhancement. However, the applied targeted metabolomics approach is suitable for a statistical differentiation between samples ${ }^{19}$ and should facilitate the identification of substances that are important for bitterness and pungency and those that are not.

Statistical Analysis. Determinations were carried out in single analyses. However, repeatability was evaluated by repetition of analysis (including extraction) of three samples for three times. The mean coefficient of variation for both methods is in all cases $<20 \%$. Correlation studies were performed using The UnscramblerX 10.1 software (Camo Inc., Oslo, Norway). Partial least-squares regression model optimization was performed based on minimization of the prediction error received by cross-validation. The presence of outliers in the sample set was evaluated after determination of the important variables. Seven samples were identified as outliers. For crossvalidation, the sample set (without the outliers) was divided randomly by the software into three groups of 16 samples. Calibrations were performed using different combinations of two of these groups. These calibrations were validated using the respective unused group. Finally, the best working model was also applied to predict bitterness and pungency of the outliers.

\section{RESULTS}

The results of the photometric determination of the total phenolic content show no satisfying correlation with the sensory evaluated bitterness $\left(R^{2}=0.58\right)$ and pungency $\left(R^{2}=\right.$
0.42) (Figure 2a,b). There are several oil samples with high total phenolic content but low bitterness/pungency intensity. Even if the correlation is conducted for olive oils from different countries separately, there is still no satisfying correlation. Correlation for olive oils from Spain $(n=18)$ results in $R^{2}=$ 0.76 , for samples from Italy $(n=15)$ results in $R^{2}=0.86$, and for samples from Portugal $(n=10)$ results in $R^{2}=0.74$. The slope $a$ of the regression lines differs strongly for the different countries (Spain, $a=0.59$; Italy, $a=1.13$; and Portugal, $a=$ 0.73). This method does not seem to be able to predict olive oil bitterness/pungency for the investigated olive oils in our study as it is described in the literature. ${ }^{12,17}$

Another method is the direct correlation of decarboxymethyl oleuropein aglycon ${ }^{9}$ or oleuropein aglycon ${ }^{16,17}$ contents with the bitterness and decarboxymethyl ligstroside aglycon content with pungency. ${ }^{18}$ Therefore, these compounds were determined semiquantitatively by a profiling approach, and the contents were correlated with the bitterness/pungency as a starting point for method refinement in our study as well. The results are shown in Figure $2 c-e$. The correlation coefficient for the pair bitterness and oleuropein aglycon is $R^{2}=0.36$, and for bitterness and decarboxymethyl oleuropein aglycon, it is $R^{2}=$ 0.25 . Both hydrophilic phenols show only a weak correlation with bitterness. Decarboxymethyl ligstroside aglycon shows a very weak correlation with pungency $\left(R^{2}=0.12\right)$. Obviously, not only a single compound in olive oil is responsible for the sensory sensations bitterness and pungency. In conclusion, to predict bitterness and pungency intensities, the contents of all secoiridoids and their derivatives must be taken into account.

Figure $2 \mathrm{f}$ shows the correlation between the sum of the contents of the secoiridoids and secoiridoid derivatives described in literature with bitterness. However, a better but 

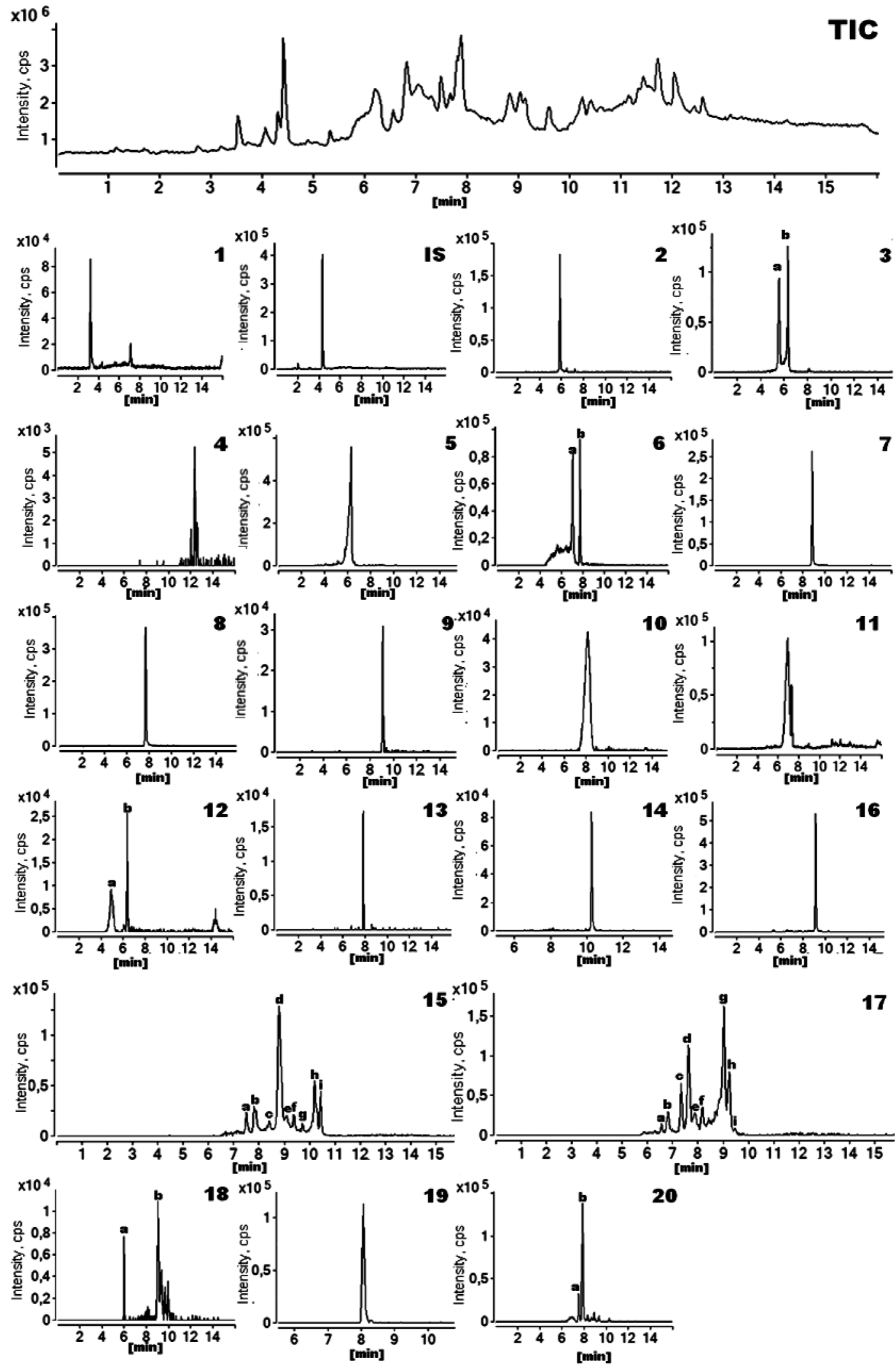

Figure 1. Total ion current (TIC) and extracted ion chromatograms $\left( \pm 10 \mathrm{ppm} ;[\mathrm{M}-\mathrm{H}]^{-}\right)$of detected hydrophilic compounds. Compound names and $m / z$ values are listed in Table 1 .

still not significant correlation $\left(R^{2}=0.46\right)$ could be obtained in this case. The reason for the poor correlation could be that there is at least one additional bitter/pungent compound in olive oil. Furthermore, the contribution of the different secoiridoids and secoiridoid derivatives to the total bitterness intensity is not identical and may vary significantly. Therefore, 
a)

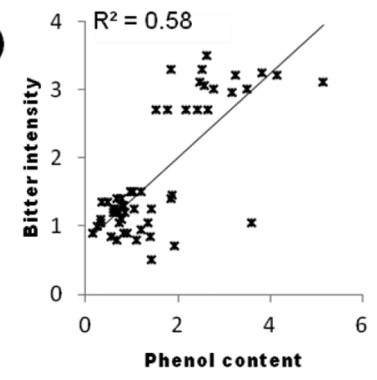

d)

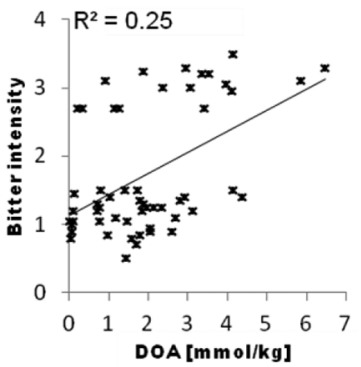

b)

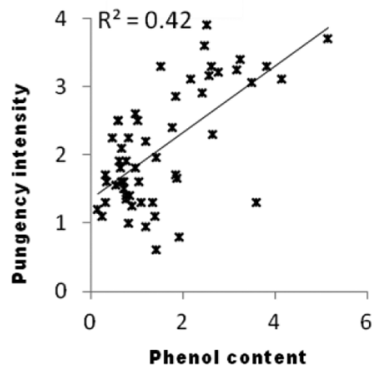

e)

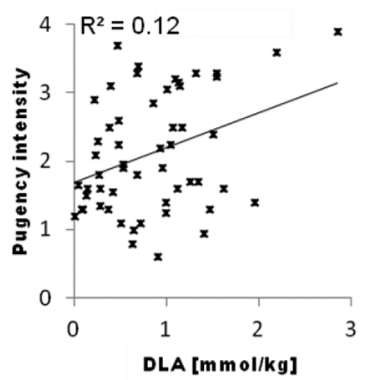

⿷)

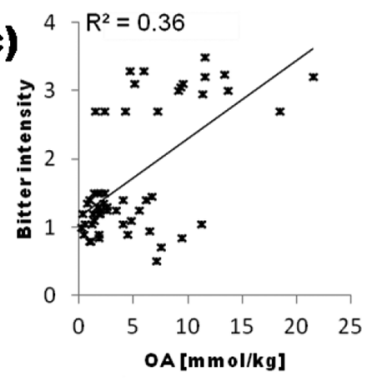

f)

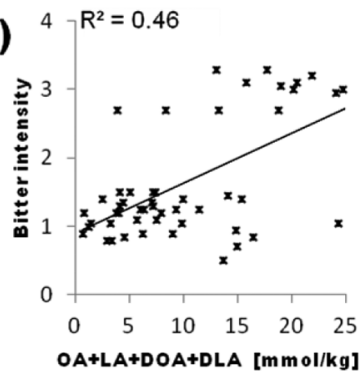

Figure 2. Correlations between instrumental measured and sensory evaluated data. OA, oleuropein aglycon; LA, ligstroside aglycon; DOA, decarboxymethyl oleuropein aglycon; and DLA, decarboxymethyl ligstroside aglycon.
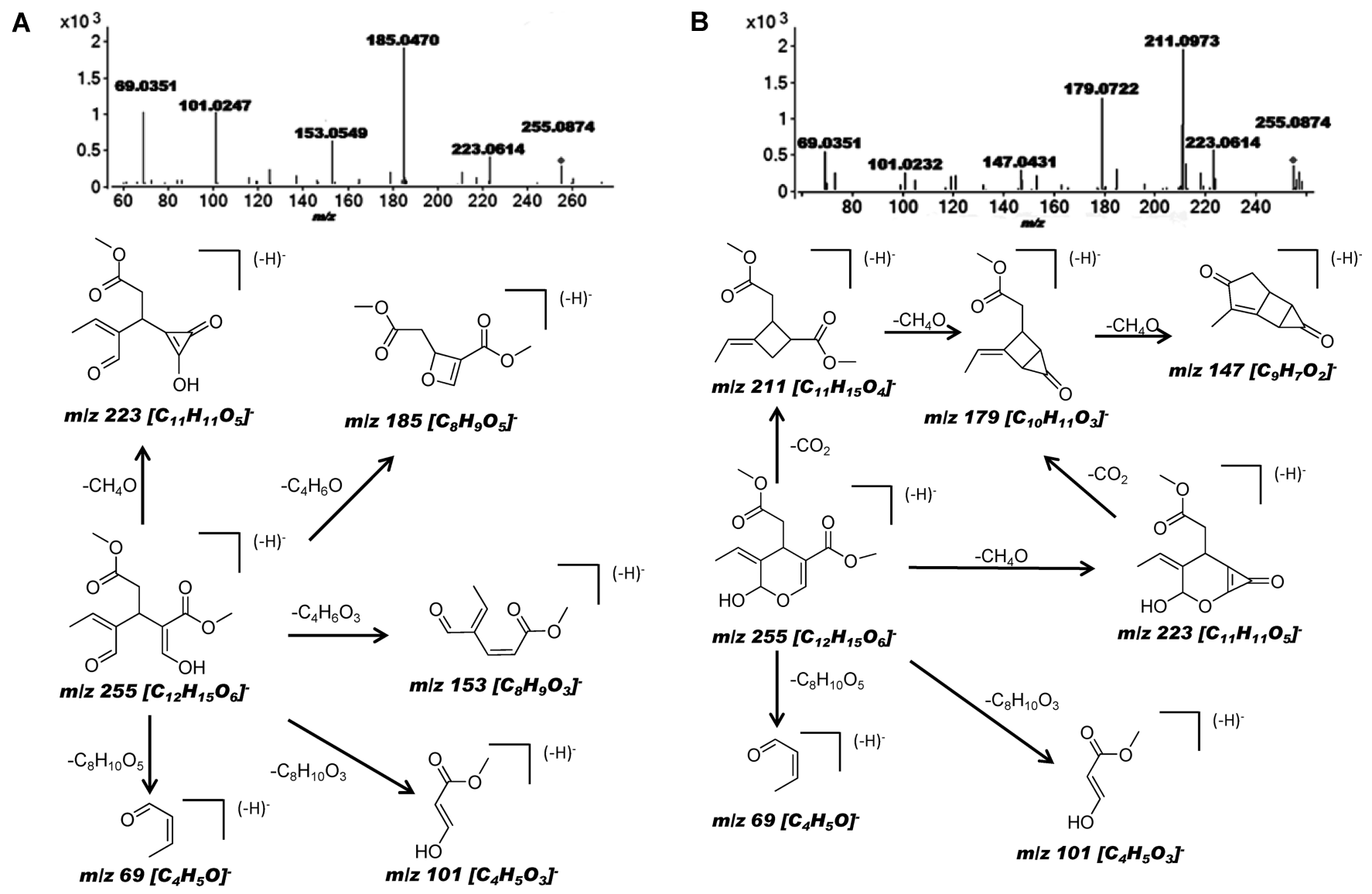

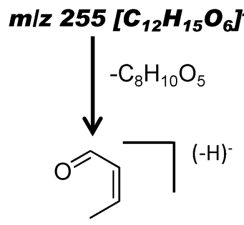

$\mathrm{m} / \mathrm{z} 69\left[\mathrm{C}_{4} \mathrm{H}_{5} \mathrm{O}\right]$

Figure 3. (A) MS/MS spectrum and possible fragmentation scheme of compound 6a (tentative elenolic acid methyl ester). The fragmentation scheme was adapted from the fragmentation scheme of ligstroside aglycone described by Fu et al. ${ }^{10}$. (B) MS/MS spectrum and possible fragmentation scheme of compound $\mathbf{6 b}$, tentative elenolic acid methyl ester (cyclic structure).

the key for successful prediction of bitterness and pungency of olive oil must be the identification of all relevant bitter/pungent compounds and the evaluation of individual weighting factors for these compounds.
As a result, we decided to establish a HPLC-MS profiling method for hydrophilic compounds responsible for the bitterness/pungency in olive oil. Extracts of 54 olive oil samples of different origin [Spain, Italy, Portugal, Israel, Greece, California (United States), France, and Turkey] were 
investigated. Table 1 shows the detected compounds. Most of the compounds were described in the literature. ${ }^{10}$ Beside the well-known secoiridoid derivatives oleuropein aglycon (nine isomers), ligstroside aglycon (nine isomers), decarboxymethyl oleuropein aglycon, and decarboxymethyl ligstroside aglycon, there are other compounds structurally related to oleuropein like methyl oleuropein (eight isomers), decarboxymethyl 10hydroxyoleuropein aglycon (two isomers), elenolic acid, and hydroxytyrosol. Compounds from other chemical classes are the flavones luteolin, apigenin, and chrysoeriol or the lignans pinoresinol and acetoxypinoresinol. Azelaic acid and myristic acid are two carboxylic acids detected in olive oil. However, there are also compounds that were not fully characterized and described in literature yet. Compound $6(\mathrm{~m} / z$ 255.0874; $[\mathrm{M}-\mathrm{H}]^{-}$) was detected but not identified by Inarejos-Garcia et al. ${ }^{14}$ Cortesi et al. detected a compound with $\mathrm{m} / \mathrm{z} 255$ by low-resolution MS, but no correlation to bitterness or pungency was established. ${ }^{20}$ In the paper, which is only available in Italian, the compound is labeled as "aglicone oleoside dimethylestere" (oleoside dimethyl ester aglycon). Although a tentative structure is displayed, no further description is given for how this assignment was made. The characterization was solely based on in-source CID without dedicated precursor ion selection and interferences of coeluting compounds are likely. In contrast, we detected two isomers and performed a detailed structural characterization by highresolution, high-mass accuracy HPLC-TOF-MS/MS and nanospray-Fourier transform ion cyclotron resonance-mass spectrometry (FTICR-MS) in multistage fragmentation mode applying precursor ion selection.

There are two isomers 6a $\left(t_{\mathrm{r}}=7.07 \mathrm{~min}, \mathrm{~m} / z 255.0874\right)$ and $\mathbf{6 b}\left(t_{\mathrm{r}}=7.75 \mathrm{~min}, \mathrm{~m} / z 255.0874\right)$ with the same accurate mass. Tentative identification was performed by calculating possible molecular formulas from accurate mass and interpretation of tandem mass spectrometric (MS/MS) data. The molecular formula calculated from $m / z 255.0874\left([\mathrm{M}-\mathrm{H}]^{-}\right)$is $\mathrm{C}_{12} \mathrm{H}_{16} \mathrm{O}_{6}$ (relative mass error $-0.1 \mathrm{ppm}$ ). MS/MS fragmentation patterns differ strongly for compounds $\mathbf{6 a}$ and $\mathbf{6 b}$ (cf. Figure 3). Fragmentation of $\mathbf{6 a}$ indicates that the unknown compound is an elenolic acid derivative, because of its similarity to fragmentation of oleuropein aglycon and ligstroside aglycon (cf. Table 2). The MS/MS spectrum and the scheme of possible fragmentation pathway adapted from the fragmenta-

Table 2. Diagnostic Fragments of MS/MS Spectra

\begin{tabular}{|c|c|c|c|c|}
\hline no. & formula & $\begin{array}{c}m / z \\
{[\mathrm{M}-\mathrm{H}]}\end{array}$ & major fragments & ref \\
\hline $3 a$ & $\mathrm{C}_{11} \mathrm{H}_{13} \mathrm{O}_{5}$ & 225.0763 & $123.04,101.02$ & \\
\hline $3 \mathbf{b}$ & $\mathrm{C}_{11} \mathrm{H}_{13} \mathrm{O}_{5}$ & 225.0768 & $193.06,181.09,149.06,121.06$ & \\
\hline 5 & $\mathrm{C}_{11} \mathrm{H}_{13} \mathrm{O}_{6}$ & 241.0718 & $\begin{array}{l}165.06,139.00,127.04,121.03 \\
\quad 101.03,95.05\end{array}$ & 25 \\
\hline $6 a$ & $\mathrm{C}_{12} \mathrm{H}_{15} \mathrm{O}_{6}$ & 255.0874 & $223.07,185.05,101.02,69.04$ & \\
\hline $6 b$ & $\mathrm{C}_{12} \mathrm{H}_{15} \mathrm{O}_{6}$ & 255.0874 & $\begin{array}{l}223.07,211.10,179.07,147.04 \\
101.03,69.04\end{array}$ & \\
\hline 10 & $\mathrm{C}_{17} \mathrm{H}_{19} \mathrm{O}_{5}$ & 303.1238 & $\begin{array}{l}285.12,183.07,179.07,165.06 \\
59.01\end{array}$ & 26 \\
\hline 11 & $\mathrm{C}_{17} \mathrm{H}_{19} \mathrm{O}_{6}$ & 319.1187 & $\begin{array}{l}249.08,195.07,183.07,139.04 \\
\quad 95.05,69.04\end{array}$ & 26 \\
\hline 15 & $\mathrm{C}_{19} \mathrm{H}_{21} \mathrm{O}_{7}$ & 361.1293 & $291.09,259.10,101.03$ & 10 \\
\hline 16 & $\mathrm{C}_{19} \mathrm{H}_{19} \mathrm{O}_{8}$ & 375.1097 & $239.05,195.07,179.03,137.03$ & \\
\hline 17 & $\mathrm{C}_{19} \mathrm{H}_{21} \mathrm{O}_{8}$ & 377.1242 & $\begin{array}{l}307.08,275.09,241.08,181.09 \\
149.02,139.00,95.05\end{array}$ & 10 \\
\hline 20 & $\mathrm{C}_{21} \mathrm{H}_{27} \mathrm{O}_{10}$ & 439.1618 & $361.13,291.09,259.10,101.03$ & \\
\hline
\end{tabular}

tion pathway of oleuropein aglycon described by $\mathrm{Fu}$ et $\mathrm{al}^{21}$ are shown in Figure 3A. The loss of one $\mathrm{CH}_{3} \mathrm{OH}$ and one $\mathrm{C}_{4} \mathrm{H}_{6} \mathrm{O}$ from $m / z 255$ yields the ions $\left[\mathrm{M}-\mathrm{H}-\mathrm{CH}_{3} \mathrm{OH}\right]^{-}(\mathrm{m} / z 223)$ and $\left[\mathrm{M}-\mathrm{H}-\mathrm{C}_{4} \mathrm{H}_{6} \mathrm{O}\right]^{-}(\mathrm{m} / z 185)$, respectively, which further form product ion $\left[\mathrm{M}-\mathrm{H}-\mathrm{C}_{4} \mathrm{H}_{6} \mathrm{O}-\mathrm{CH}_{3} \mathrm{OH}\right]^{-}(\mathrm{m} / z$ 153). The fragment $m / z 125$ results from the loss of $\mathrm{CH}_{3} \mathrm{OCO}$ from $\left[\mathrm{M}-\mathrm{H}-\mathrm{C}_{4} \mathrm{H}_{6} \mathrm{O}\right]^{-}(\mathrm{m} / z$ 185). Compound 6a could be tentatively identified as elenolic acid methyl ester based on these results. This cyclic form is in accordance with the structure proposed by Cortesi et al. ${ }^{20}$

It is assumed that $\mathbf{6 b}$ is also an isomer of elenolic acid methyl ester, although the fragmentation pattern differs strongly from that of 6a. This assumption is based on the observance of interconversion of one isomer into the other between different analyses of the same olive oil sample. The MS/MS spectrum and the scheme of possible fragmentation are shown in Figure $3 \mathrm{~B}$. The loss of one $\mathrm{CH}_{3} \mathrm{OH}$ and one $\mathrm{CO}_{2}$ from $\mathrm{m} / z 255$ yields the ions $\left[\mathrm{M}-\mathrm{H}-\mathrm{CH}_{3} \mathrm{OH}\right]^{-}(m / z 223)$ and $\left[\mathrm{M}-\mathrm{H}-\mathrm{CO}_{2}\right]^{-}$ $(\mathrm{m} / z 211)$, respectively, which further form product ion $[\mathrm{M}-$ $\left.\mathrm{H}-\mathrm{CO}_{2}-\mathrm{CH}_{3} \mathrm{OH}\right]^{-}(\mathrm{m} / z$ 179). The fragment $m / z 147$ results from the loss of $\mathrm{CH}_{4} \mathrm{O}$ from $\left[\mathrm{M}-\mathrm{H}-\mathrm{CO}_{2}-\mathrm{CH}_{3} \mathrm{OH}\right]^{-}(\mathrm{m} / z$ 179). The loss of $\mathrm{CO}_{2}$ indicates that $\mathbf{6 b}$ is the cyclic structure of elenolic acid methyl ester, while $\mathbf{6 a}$ is the open-ring structure. This open-ring structure was not detected by Cortesi et al. ${ }^{20}$

Confirmation of the assigned sum formulas was performed by FTICR-MS ${ }^{n}$ experiments. The respective data are presented in the Supporting Information. The identification is tentative and has to be verified by additional investigations (e.g., NMR experiments). Nevertheless, the MS/MS fragmentation patterns show that there is no phenolic substructure in the molecules. Hence, these components are not detected by photometric total phenolic content determination.

Compound $3\left(\mathrm{~m} / z\right.$ 225; $\left.[\mathrm{M}-\mathrm{H}]^{-}\right)$was detected but not identified by Carrasco-Pancorbo et al. ${ }^{6}$ Two isomers 3a $\left(t_{\mathrm{r}}=\right.$ $5.55 \mathrm{~min}, \mathrm{~m} / z 225.0763)$ and $3 \mathbf{b}\left(t_{\mathrm{r}}=6.31 \mathrm{~min}, \mathrm{~m} / z 225.0768\right)$ could be separated by HPLC and have a calculated molecular formula of $\mathrm{C}_{11} \mathrm{H}_{14} \mathrm{O}_{5}$ (relative mass error -2.4 and $0.2 \mathrm{ppm}$, respectively). MS/MS spectra of these isomers (cf. Supporting Information, Figures S1 and S2) differ also from each other. The fragmentation pattern of compound $\mathbf{3 b}$ is very similar to fragmentation of $6 \mathbf{b}$, but the fragments show a mass difference of $30 \mathrm{Da}$ to those of compound $\mathbf{6 b}$. The losses of $\mathrm{CH}_{3} \mathrm{OH}$ and $\mathrm{CO}_{2}$ from $\mathrm{m} / z 225$ yield the ions $\left[\mathrm{M}-\mathrm{H}-\mathrm{CH}_{3} \mathrm{OH}\right]^{-}(\mathrm{m} / z$ $193)$ and $\left[\mathrm{M}-\mathrm{H}-\mathrm{CO}_{2}\right]^{-}(m / z 181)$, respectively, which further form product ion $\left[\mathrm{M}-\mathrm{H}-\mathrm{CO}_{2}-\mathrm{CH}_{3} \mathrm{OH}\right]^{-}(\mathrm{m} / z$ 149). The fragment $m / z 121$ results from the loss of $\mathrm{CO}$ from $\left[\mathrm{M}-\mathrm{H}-\mathrm{CO}_{2}-\mathrm{CH}_{3} \mathrm{OH}\right]^{-}(\mathrm{m} / z$ 149). A possible structure, which fits well to the fragmentation pattern, is the cyclic structure of elenolic aldehyde [4-formyl-2-(hydroxymethylene)3-(2-oxoethyl)-4-hexenoic acid methyl ester]. The two fragments $\left[\mathrm{M}-\mathrm{H}-\mathrm{C}_{4} \mathrm{H}_{6} \mathrm{O}_{3}\right]^{-}(m / z \quad 123)$ and $[\mathrm{M}-\mathrm{H}-$ $\left.\mathrm{C}_{7} \mathrm{H}_{8} \mathrm{O}_{2}\right]^{-}(\mathrm{m} / z$ 101) of $3 \mathrm{a}$ can be explained by the openring structure of elenolic aldehyde.

For compound $16\left(\mathrm{~m} / z\right.$ 375.1097; $\left.[\mathrm{M}-\mathrm{H}]^{-}\right)$the molecular formula $\mathrm{C}_{19} \mathrm{H}_{20} \mathrm{O}_{8}$ (relative mass error $-3.1 \mathrm{ppm}$ ) was calculated. In addition, the MS/MS fragments $(m / z 239,179$, and 137), which are $2 \mathrm{Da}$ lower than the corresponding fragments of oleuropein aglycon $17(\mathrm{~m} / z 241,181$, and 139), indicate that the compound is a dehydro derivative of oleuropein aglycon. The same considerations result for compound $14\left(\mathrm{~m} / z\right.$ 359.1144; $\left.[\mathrm{M}-\mathrm{H}]^{-}\right)$with the molecular formula $\mathrm{C}_{19} \mathrm{H}_{20} \mathrm{O}_{7}$ (relative mass error $-2.1 \mathrm{ppm}$ ), which corresponds to a dehydro derivative of ligstroside aglycon. 
Compound $20(m / z 439.1618)$ has the molecular formula $\mathrm{C}_{21} \mathrm{H}_{28} \mathrm{O}_{10}$ (relative mass error $-1.9 \mathrm{ppm}$ ). The fragmentation pattern of compound $20(\mathrm{~m} / z 361,291,259,101)$ shows high similarity to the fragmentation of ligstroside aglycone $(\mathrm{m} / z$ $361,291,259,101)$. This and the fact that the two isomers 20a and $20 \mathrm{~b}$ have the same retention time $\left(t_{\mathrm{r}}=7.50 \mathrm{~min}\right.$ and $t_{\mathrm{r}}=$ $7.88 \mathrm{~min}$ ) as the isomers $\mathbf{1 5 a}$ and $\mathbf{1 5 b}$ ligstroside aglycon indicate that compound $\mathbf{2 0}$ is an adduct with methanol $\left(\mathrm{CH}_{3} \mathrm{OH}, 32 \mathrm{Da}\right)$ and formate $\left(\mathrm{HCOO}^{-}, 45 \mathrm{Da}\right)$, which is formed as artifact during ionization.

Following the identification of possible phenolic compounds, semiquantitative data of the 25 identified compounds were correlated with sensory data by partial least-squares regression (PLS-R) to determine the influence of the different compounds on olive oil bitterness and pungency. In Figure 4, the

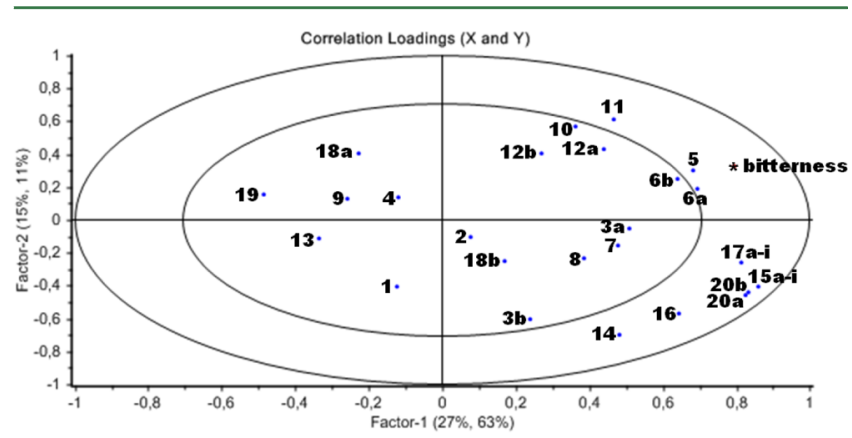

Figure 4. Correlation loadings plot of PLS-R model. $X$ variables were semiquantitative data of all 25 compounds, and the $Y$ variable was the bitterness intensity evaluated by sensoric panel.

correlation loadings plot of the PLS-R model for bitterness is shown. Variables between the inner and the outer ring are highly correlated with principal component 1 (PC1) and/or PC2. Bitterness is highly positively correlated with PC1 $(R=$ $0.79)$ and weakly positively correlated to PC2 $(R=0.33)$. Table 3 shows the correlation coefficients for PC1 and PC2. Compounds $3 b, 5,6 a, 6 b, 10,11,12 a, 14,15 a-i, 16,17 a-i$, 20a, and 20b show a strong positive correlation with bitterness, whereas compounds 1, 4, 9, 13, 18a, and 19 show a negative correlation with bitterness. Compounds 2, 3a, 7, 8, 12b, and $18 \mathrm{~b}$ were only weakly positively correlated with bitterness. Variables 6a, 6b, 15a-i, 20a, and 20b are located closely

Table 3. Correlation Coefficients Obtained between Sensory Evaluated Bitterness and Semiquantitative Data by Partial Least Square Regression (PLS-R)

\begin{tabular}{lrrlrr} 
variable & PC 1 & PC2 & variable & PC 1 & \multicolumn{1}{c}{ PC2 } \\
bitterness & 0.793 & 0.328 & $\mathbf{1 1}$ & 0.467 & 0.609 \\
$\mathbf{1}$ & -0.124 & -0.409 & $\mathbf{1 2 a}$ & 0.439 & 0.432 \\
$\mathbf{2}$ & 0.076 & -0.105 & $\mathbf{1 2 b}$ & 0.269 & 0.409 \\
$\mathbf{3 a}$ & 0.508 & -0.054 & $\mathbf{1 3}$ & -0.337 & -0.113 \\
$\mathbf{3 b}$ & 0.238 & -0.606 & $\mathbf{1 4}$ & 0.484 & -0.696 \\
$\mathbf{4}$ & -0.118 & 0.142 & $\mathbf{1 5 a}-\mathbf{i}$ & 0.862 & -0.407 \\
$\mathbf{5}$ & 0.684 & 0.304 & $\mathbf{1 6}$ & 0.645 & -0.569 \\
$\mathbf{6 a}$ & 0.695 & 0.189 & $\mathbf{1 7 a}-\mathbf{i}$ & 0.812 & -0.257 \\
$\mathbf{6 b}$ & 0.639 & 0.249 & $\mathbf{1 8 a}$ & -0.228 & 0.405 \\
$\mathbf{7}$ & 0.478 & -0.156 & $\mathbf{1 8 b}$ & 0.169 & -0.252 \\
$\mathbf{8}$ & 0.386 & -0.230 & $\mathbf{1 9}$ & -0.486 & 0.159 \\
$\mathbf{9}$ & -0.259 & 0.127 & $\mathbf{2 0 a}$ & 0.827 & -0.456 \\
$\mathbf{1 0}$ & 0.364 & 0.574 & $\mathbf{2 0 b}$ & 0.832 & -0.442
\end{tabular}

together in the correlation loadings plot. Therefore, these variables have the same correlation with bitterness. Isomers 6 a and $\mathbf{6 b}$ have the same bitter intensity. Compounds 20a and $20 \mathrm{~b}$ are adducts of $\mathbf{1 5 a}$ and $\mathbf{1 5 b}$; hence, it is not surprising that these variables show the same correlation with bitterness. The results for pungency are similar (cf. Table 4). The compounds

Table 4. Correlation Coefficients Obtained between Sensory Evaluated Pungency and Semiquantitative Data by Partial Least Square Regression (PLS-R)

\begin{tabular}{lrrlrr} 
variable & PC 1 & PC2 & variable & PC 1 & \multicolumn{1}{c}{ PC2 } \\
pungency & 0.730 & 0.380 & $\mathbf{1 1}$ & 0.518 & 0.494 \\
$\mathbf{1}$ & -0.166 & -0.388 & $\mathbf{1 2 a}$ & 0.496 & 0.327 \\
$\mathbf{2}$ & 0.034 & -0.180 & $\mathbf{1 2 b}$ & 0.324 & 0.311 \\
$\mathbf{3 a}$ & 0.496 & -0.118 & $\mathbf{1 3}$ & -0.347 & -0.038 \\
$\mathbf{3 b}$ & 0.175 & -0.565 & $\mathbf{1 4}$ & 0.386 & -0.724 \\
$\mathbf{4}$ & -0.093 & 0.181 & $\mathbf{1 5 a}-\mathbf{i}$ & 0.786 & -0.545 \\
$\mathbf{5}$ & 0.714 & 0.209 & $\mathbf{1 6}$ & 0.559 & -0.642 \\
$\mathbf{6 a}$ & 0.723 & 0.124 & $\mathbf{1 7 a}-\mathbf{i}$ & 0.750 & -0.425 \\
$\mathbf{6 b}$ & 0.682 & 0.219 & $\mathbf{1 8 a}$ & -0.129 & 0.495 \\
$\mathbf{7}$ & 0.474 & -0.146 & $\mathbf{1 8 b}$ & 0.133 & -0.280 \\
$\mathbf{8}$ & 0.381 & -0.188 & $\mathbf{1 9}$ & -0.437 & 0.260 \\
$\mathbf{9}$ & -0.205 & 0.249 & $\mathbf{2 0 a}$ & 0.750 & -0.571 \\
$\mathbf{1 0}$ & 0.426 & 0.432 & $\mathbf{2 0 b}$ & 0.759 & -0.552 \\
\hline
\end{tabular}

that show a high correlation with bitterness and pungency should be mainly responsible for the bitter and pungent taste. After identification of these compounds, a multivariate regression model has to be evaluated using individual weighting factors for each compound, which takes into account the different bitterness and pungency intensities. Consequently, further regression models were computed using only compounds, which show a strong positive correlation with bitterness. The sums of the highly correlated isomers $\mathbf{6 a}, \mathbf{6 b}$, and the ligstroside aglycon species $15 a-i, 20 a$, and 20b were used to reduce not relevant variation. Model optimization was performed by reducing the error of cross-validation (66\% of the samples were used for calibration and $33 \%$ for validation). Best results were obtained using compounds $5, \Sigma(6 \mathbf{a}, 6 \mathbf{b}), 10,11$, $\Sigma(15 a-i, 20 a, 20 b)$, and $17 a-i$ as variables. In Figure $5 a$, the results of the best performing PLS-R model [two PCs, $R^{2}=0.8$ (predicted vs measured), standard error cross-validation SECV $=0.3]$ are depicted. The predicted bitterness of the samples is plotted against sensory evaluated bitterness. These prediction results were obtained when the samples were used for validation. The model is able to predict the bitterness for most olive oils in the error margin of the sensory evaluation of \pm 0.5 . Seven olive oil samples (marked with symbol + ) were identified as outliers and left out for calibration, because the prediction error for these samples was always higher than for the other samples. The reasons for these discrepancies are not clear, but four of these samples had an off-flavor (fusty or wet wood), which may have hampered sensory evaluation of bitterness. Weighted regression coefficients are listed in Table 5 , and coefficients are similar for all six compounds. Elenolic acid methyl ester 6 and the secoiridoide oleuropein aglycon $17 \mathbf{a}-\mathbf{i}$ and ligstroside aglycon $\Sigma(15 \mathbf{a}-\mathbf{i}, 20 \mathbf{a}, 20 \mathbf{b})$ have the greatest influence on bitterness.

The same variables as for bitterness gave best results for prediction of pungency, but the results were inferior to those for bitterness (Figure $5 \mathrm{~b}$ ). Regression coefficients of the best PLS-R model [one PC, $R^{2}=0.7$ (predicted vs measured), 

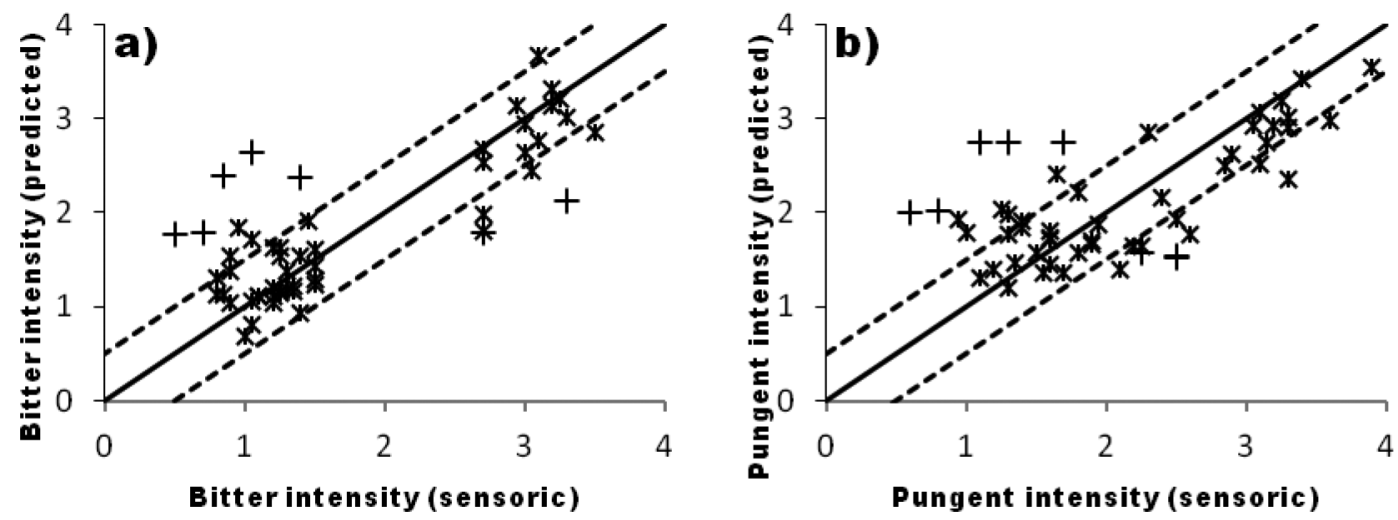

Figure 5. Results for prediction of (a) bitterness and (b) pungency. All results from cross-validation (67\% of samples for calibration and $33 \%$ for validation). Samples represented by the symbol cross $(+)$ were not used for calibration. Continuous line represents perfect linear correlation; broken lines represent the error margin of sensory results $( \pm 0.5)$.

Table 5. Weighted Regression Coefficients of PLS-R

\begin{tabular}{ccccccccc} 
& B0 & $\mathbf{5}$ & $\Sigma(\mathbf{6 a}, \mathbf{b})$ & $\mathbf{1 0}$ & $\mathbf{1 1}$ & $\Sigma(\mathbf{1 5 a}-\mathbf{i}, \mathbf{2 0 a}, \mathbf{b})$ & $\mathbf{1 7 a}-\mathbf{i}$ & PCs \\
\hline bitterness & 0.63 & 0.15 & 0.31 & 0.05 & 0.11 & 0.22 & 0.22 & 2 \\
pungency & 1.05 & 0.19 & 0.22 & 0.11 & 0.15 & 0.16 & 0.16 & 1
\end{tabular}

standard error cross-validation SECV $=0.4]$ for pungency are similar to the coefficients for bitterness, whereas decarboxymethyl oleuropein aglycon $\mathbf{1 1}$ and decarboxymethyl ligstroside aglycon 10 have a higher influence on pungency than on bitterness.

\section{DISCUSSION}

No correlation between the total phenolic compound content and the bitterness/pungency could be found. Evaluation of bitterness and pungency of olive oil by determination of the total phenol content is based on the assumption that all bitter and pungent compounds in olive oil are phenols or that their contents correlate with those of total phenolic compounds. The pattern of the different phenolic compounds in olive oil depends on variety and origin. Consequently, evaluation of bitterness by total phenol compounds content could only work with separate regression models for each variety and region of origin. This is indicated by the results of Mateos et al., ${ }^{16}$ who found regression lines with different slopes for different varieties from Spain. In a similar way, a difference in the slopes of the regression lines for olive oil from Spain, Italy, and Portugal was observed in this work. Mateos et al. ${ }^{16}$ found only poor correlation between total phenol content and bitterness for some varieties. This is in agreement with the findings by Angerosa et al. ${ }^{1}$ and the results of this work. The nonphenolic compounds elenolic acid and elenolic acid methyl ester have to be considered in evaluating bitterness/pungency. In conclusion, an evaluation of bitterness/pungency of olive oil could not be performed by measurement of total phenolic compounds content alone, because elenolic acid and elenolic acid methyl ester would not be detected.

Several studies dealing with the evaluation of bitterness/ pungency of olive oil by HPLC methods have been described: Guitiérrez-Rosales et al. determined the content of oleuropein aglycon, ligstroside aglycon, decarboxymethyl oleuropein aglycon, and decarboxymethyl ligstroside aglycon in 20 olive oils by HPLC-UV $(280 \mathrm{~nm}) .^{9}$ They obtained best correlation between the content of decarboxymethyl oleuropein aglycon and decarboxymethyl ligstroside aglycon with bitterness intensity. Correlation was described by a linear function.

In a similar way, Mateos et al. used linear functions to describe correlations between quantification data of oleuropein aglycon, ligstroside aglycon, decarboxymethyl oleuropein aglycon, and decarboxymethyl ligstroside aglycon. ${ }^{16}$ Interestingly, they came to the conclusion that oleuropein aglycon content showed the best correlation with bitterness intensity.

Siliani et al. found also the best correlation between oleuropein aglycon content and bitterness intensity. However, they used an exponential regression model for correlation. ${ }^{17}$ Correlation between pungency intensity and content of these compounds were investigated by none of the authors. Andrewes et al. described decarboxymethyl ligstroside aglycon as "a key contributor to pungency". ${ }^{18}$ The authors fractionated the ethanol/water $(60: 40)$ extract of olive oil by preparative HPLC. The fractions were sensory evaluated. The fraction containing decarboxymethyl ligstroside aglycon caused a strong burning sensation. However, a correlation between decarboxymethyl ligstroside aglycon concentration in different olive oils and their pungency intensity was not performed.

Correlations between one of these compounds and the bitter/pungent taste of olive oil could not be observed in our study. Contribution of the hydrophilic compounds in olive oil depends on variety and geographical origin of the olive fruits. ${ }^{22}$ Hence, there should be no overall correlation between the different compounds. Because of this, evaluation of bitterness/ pungency by quantification of a single compound could not give satisfactory results. Guitiérrez et al. set up a multiple linear regression using peak areas of four not identified peaks (HPLCUV, $225 \mathrm{~nm}) .^{15}$ However, the limited number of only 10 olive oils and the absence of method validation show the preliminary character of this study.

In this work, the influence of 25 compounds detected in 54 olive oils was evaluated by correlation of semiquantitative data obtained by the profiling approach with sensory results. The best correlations show oleuropein aglycon 17 (sum of isomers), ligstroside aglycon 15, 20 (sum of isomers), decarboxymethyl oleuropein aglycon 11, decarboxymethyl ligstroside aglycon 10, 
elenolic acid 5, and elenolic acid methyl ester 6 (sum of isomers). A PLS-R model to predict bitterness and pungency of olive oil using contents of these six compounds could be set up. Compounds 10, 11, 15, and 17 are well-known phenolic compounds, which were used for prediction of bitterness in several studies. However, contrary to the methods described in the literature, in this work, a multivariate regression model was set up. The different influences of the phenolic compounds on the bitterness and pungency are taken into account by using individual regression coefficients for each compound. In addition to the phenolic compounds, the nonphenolic elenolic acid and elenolic acid methyl ester show high correlation to bitterness and pungency. However, sensory attributes of purified compounds still have to be evaluated. For this reason, it is not clear whether the compounds cause a bitter and/or a pungent taste or not. Mateos et al. evaluated the bitter taste of components of the phenolic fraction of olive oil. ${ }^{16}$ Elenolic acid showed no bitter taste in a concentration of approximately 0.25 $\mathrm{mmol} / \mathrm{L}$. However, elenolic acid was detected in even higher concentrations (median $2.2 \mathrm{mmol} / \mathrm{kg}$ olive oil) in this work. Furthermore, elenolic acid was obtained from oleuropein by hydrolysis with $0.5 \mathrm{~mol} / \mathrm{L}$ sulfuric acid at $55^{\circ} \mathrm{C}$, and thus, a transformation into a not bitter tasting isomer could have occurred. Oleuropein aglycon showed a high intensity of bitterness even in small concentrations $(0.05 \mathrm{mmol} / \mathrm{L})$. In further investigations the six compounds should be isolated from olive oil to evaluate their bitterness (and pungency).

No clear differentiation between the bitter and the pungent taste could be found in the distribution of the studied hydrophilic compounds. Sensory data of bitterness and pungency show a high colinearity caused by the fact that both sensations are generated in the same gustative papillae. $^{23,24}$ However, there are oils that show significant differences in bitterness and pungency intensity, and especially these oils show high differences between predicted and sensory evaluated pungency. This suggests that there is at least one more compound causing only one of the sensory attributes. Therefore, future investigations will focus on the identification and characterization of these compounds in more nonpolar fractions of olive oils.

\section{ASSOCIATED CONTENT}

\section{S Supporting Information}

Semiquantitative data of 25 analytes and sensory evaluated data for all 54 olive oils. MS/MS spectra and possible fragmentation pathway of compounds $\mathbf{3 a}$ and $\mathbf{3 b}$. Complementary structural elucidation of compounds $\mathbf{6 a}$ and $\mathbf{6 b}$ by nanospray-Fourier transform ion cyclotron resonance-mass spectrometry (FTICR-MS) in multistage fragmentation mode. This material is available free of charge via the Internet at http://pubs.acs.org.

\section{AUTHOR INFORMATION}

\section{Corresponding Author}

*Tel: (+49)202 4393457. Fax: (+49)202 4393073. E-mail: hayen@uni-wuppertal.de.

\section{Present Address}

"Institute for Prevention and Occupational Medicine of the German Social Accident Insurance, Institute of the RuhrUniversität Bochum (IPA), Bochum, Germany.

Notes

The authors declare no competing financial interest.

\section{ACKNOWLEDGMENTS}

The authors are grateful to the Swiss Olive Oil Panel for the sensory evaluation of samples. We acknowledge Helma Geltenpoth (Leibniz-Institut für Analytische Wissenschaften, ISAS, e.V, Dortmund, Germany) for the skilled technical assistance in nanospray-FTICR-MS measurements.

\section{ABBREVIATIONS USED}

PLS-R, partial least-squares regression; HPLC-MS/MS, highperformance liquid chromatography-tandem mass spectrometry

\section{REFERENCES}

(1) Angerosa, F.; Mostallino, R.; Basti, C.; Vito, R. Virgin olive oil odour notes: Their relationships with volatile compounds from the lipoxygenase pathway and secoiridoid compounds. Food Chem. 2000, $68,283-287$.

(2) El Riachy, M.; Priego-Capote, F.; León, L.; Rallo, L.; de Luque Castro, M. D. Hydrophilic antioxidants of virgin olive oil. Part 1: Hydrophilic phenols: A key factor for virgin olive oil quality. Eur. J. Lipid Sci. Technol. 2011, 113, 678-691.

(3) Revised method for the organoleptic assessment of virgin olive oil, Decision DEC-21/95-V/2007, Madrid, Spain, 2007.

(4) García-González, D. L.; Aparicio, R. Research in olive oil: Challenges for the near future. J. Agric. Food Chem. 2010, 58, 1256912577.

(5) Bianco, A.; Buiarelli, F.; Cartoni, G.; Coccioli, F.; Jasionowska, R.; Margherita, P. Analysis by liquid chromatography-tandem mass spectrometry of biophenolic compounds in virgin olive oil, Part II. J. Sep. Sci. 2003, 26, 417-424.

(6) Carrasco-Pancorbo, A.; Neusüß, C.; Pelzing, M.; SeguraCarretero, A. Fernández-Gutiérrez, A. CE- and HPLC-TOF-MS for the characterization of phenolic compounds in olive oil. Electrophoresis 2007, 28, 806-821.

(7) Caruso, D.; Colombo, R.; Patelli, R.; Giavarini, F.; Galli, G. Rapid evaluation of phenolic component profile and analysis of oleuropein aglycon in olive oil by atmospheric pressure chemical ionization-mass spectrometry (APCI-MS). J. Agric. Food Chem. 2000, 48, 1182-1185.

(8) Christophoridou, S.; Dais, P.; Tseng, L.-H.; Spraul, M. Separation and identification of phenolic compounds in olive oil by coupling highperformance liquid chromatography with postcolumn solid-phase extraction to nuclear magnetic resonance spectroscopy (LC-SPENMR). Anal. Chim. Acta 2005, 53, 4667-4679.

(9) Gutiérrez-Rosales, F.; Ríos, J. J.; Gómez-Rey, M. L. Main polyphenols in the bitter taste of virgin olive oil. Structural confirmation by on-line high-performance liquid chromatography electrospray ionization mass spectrometry. J. Agric. Food Chem. 2003, $51,6021-6025$.

(10) Fu, S.; Segura-Carretero, A.; Arráez-Román, D.; Menéndez, J. A.; La Torre, A. de; Fernández-Gutiérrez, A. Tentative characterization of novel phenolic compounds in extra virgin olive oils by rapidresolution liquid chromatography coupled with mass spectrometry. J. Agric. Food Chem. 2009, 57, 11140-11147.

(11) Gutiérrez Rosales, F.; Perdiguero, S.; Gutiérrez, R.; Olias, J. Evaluation of the bitter taste in virgin olive oil. J. Am. Oil Chem. Soc. 1992, 69, 394-395.

(12) Beltrán, G.; Ruano, M. T.; Jiménez, A.; Uceda, M.; Aguilera, M. P. Evaluation of virgin olive oil bitterness by total phenol content analysis. Eur. J. Lipid Sci. Technol. 2007, 109, 193-197.

(13) Mateos, R.; Garcia-Ortiz Civantos, C.; Castro, J.; Garcia-Mesa, J. A. Direct spectrophotometric determination of bitterness in virgin olive oil without prior isolation by $\mathrm{pH}$ gradient. J. Agric. Food Chem. 2005, 53, 9615-9619.

(14) Inarejos-Garcia, A. M.; Androulaki, A.; Salvador, M. D.; Fregapane, G.; Tsimidou, M. Z. Discussion on the objective evaluation of virgin olive oil bitterness. Food Res. Int. 2009, 42, 279-284. 
(15) Gutiérrez, F.; Albi, M. A.; Palma, R.; Rios, J. J.; Olias, J. M. Bitter taste of virgin olive oil: Correlation of sensory evaluation and instrumental HPLC analysis. J. Food Sci. 1989, 54, 68-70.

(16) Mateos, R.; Cert, A.; Pérez-Camino, M.; García, J. Evaluation of virgin olive oil bitterness by quantification of secoiridoid derivatives. $J$. Am. Oil Chem. Soc. 2004, 81, 71-75.

(17) Siliani, S.; Mattei, A.; Innocenti, L. B.; Zanoni, B. Bitter taste and phenolic compounds in extra virgin olive oil: An empirical relationship. J. Food Qual. 2006, 29, 431-441.

(18) Andrewes, P.; Busch, J. L. H. C.; Joode, T.; de; Groenewegen, A.; Alexandre, H. Sensory Properties of Virgin Olive Oil Polyphenols: Identification of deacetoxy-ligstroside aglycon as a key contributor to pungency. J. Agric. Food Chem. 2003, 51, 1415-1420.

(19) Cevallos-Cevallos, J. M.; Reyes-De-Corcuera, J. I.; Etxeberria, E.; Danyluk, M. D.; Rodrick, G. E. Metabolomic analysis in food science: a review. Trends Food Sci. Technol. 2009, 20, 557-566.

(20) Cortesi, N.; Azzolini, M.; Rovellini, P.; Fedeli, E. I componenti minori polari degli oli vergini di oliva: Ipotesi di struttura mediante LC-MS. Riv. Ital. Sostanze Grasse 1995, 72, 241-251.

(21) Fu, S.; Arráez-Román, D.; Menéndez, J. A.; Segura-Carretero, A.; Fernández-Gutiérrez, A. Characterization of isomers of oleuropein aglycon in olive oils by rapid-resolution liquid chromatography coupled to electrospray time-of-flight and ion trap tandem mass spectrometry. Rapid Commun. Mass Spectrom. 2009, 23, 51-59.

(22) Ouni, Y.; Taamalli, A.; Gómez-Caravaca, A. M.; SeguraCarretero, A.; Fernández-Gutiérrez, A.; Zarrouk, M. Characterisation and quantification of phenolic compounds of extra-virgin olive oils according to their geographical origin by a rapid and resolutive LCESI-TOF MS method. Food Chem. 2011, 127, 1263-1267.

(23) Whitehead, M. C.; Beeman, C. S.; Kinsella, B. A. Distribution of taste and general sensory nerve endings in fungiform papillae of the hamster. Am. J. Anat. 1985, 173, 185-201.

(24) Bate-Smith, E. C. Haemanalysis of tannins: The concept of relative astringency. Phytochemistry 1973, 12, 907-912.

(25) Bazoti, F. N.; Gikas, E.; Tsarbopoulos, A. Simultaneous quantification of oleuropein and its metabolites in rat plasma by liquid chromatography electrospray ionization tandem mass spectrometry. Biomed. Chromatogr. 2010, 24, 506-515.

(26) Obied, H. K.; Bedgood, D.; Prenzler, P.; Robards, K. Chemical screening of olive biophenol extracts by hyphenated liquid chromatography. Anal. Chim. Acta 2007, 603, 176-189. 